\title{
Experiências em Buscas de Informações por Residentes de Medicina ${ }^{1}$
}

\author{
Experience with Information Searches by \\ Medical Residents
}

Helena Maria da Costa Lima

PNAVRASOHALE:

- Educação Médica;

Internato e Residência;

- Biblicotecas Médicas;

- Aprendizagem Baseada en Problemas.

KEY.WORIS:

Education, Medical;

Internship and residency:

Libraries, Medical.

Problemz-Based Learning.

Reccbido en: 07/07/2004

Recucaminhado em: 09/11/2004

Aprovado em: 06/12/2004

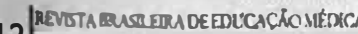

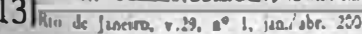

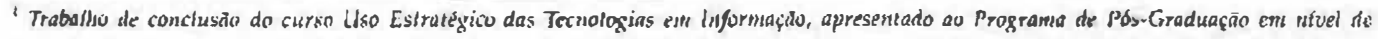

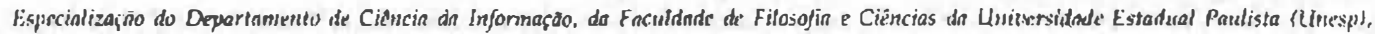
Campus de Mistilin. 


\section{INTRODUÇÃO}

Neste estudo, pretende-se investigar quais sảo as habilidades em buscas de informações e uso de bibliotecas, adquiridas durante a graduação por um grupo de profissionais médicos recém-formados - os residentes em Medicina da Faculdade de Medicina de Marília (Famema) -, que ainda năo estão no mercado de trabalho propriamente dito, mas se preparando para isto.

Os residentes constituem um grupo de usuários valioso para este estudo, uma vez que são oriundos de variadas escolas de Medicina no Brasil, com realidades e experiências diferentes, o que permite tentar conhecer um pouco de suas experiências em uso de bibliotecas e informaçōes durante a graduação. Outro fator importante é que o grupo é composto por médicos formados pelas metodologias de ensino médico tradicional e ABP (aprendizagem baseada em problemas), 0 que nos possibilita verificar se existem diferenças ou não entre as mesmas quanto às habilidades em buscas de informaçōes.

De modo geral, a Educação Médica no Brasil, até recentemente, esteve voltada à doença, à departamentalização do ensino, ao ensino centrado no professor como transmissor do conhecimento que julga ser necessário ao aluno, à especialização médica por áreas ou subáreas e ao uso de novos recursos tecnológicos, principalmente para diagnóstico. Paralelamente a esse cenário, surgiram tentativas de melhorias e adequação do ensino médico brasileiro ao longo dos anos'.

Atualmente, os programas de formação profissional devem trabalhar de forma integrada, um apoiando o outro, criando espaços de aprendizagem experimentais, onde os estudantes não sejam apenas observadores ${ }^{2}$.

Segundo Lima-Gonçalves ${ }^{3}$, tem sido um desafio para as escolas médicas definir os conhecimentos, habilidades, atitudes e valores que deverāo compor o profissional médico. $O$ mesmo autor afirma que o médico do século 21 deverá reconhecer que, para o bom desempenho profissional, precisará ser um eterno estudante, sempre carente de inforınaçðes e de procedimentos, e que a educação durante a graduação deverá ser apenas o começo de um aprendizado a se desenvolver ao longo da vida. A graduação terá o papel de encorajar o estudante a assumir a responsabilidade pelo próprio aprendizado.

Como é relatado em ampla literatura, as universidades e as profissões, de forma geral, vêem-se atingidas diretamente pela revolução tecnológica. $O$ acesso à informação tornou-se mais fácil e mais rápido, a informaçăo está mais disponivel. A preocupação atual não deve se restringir a passar informaçōes, deve-se também mostrar como o indivíduo ou a comunidade poderăo usar, selecionar, manipular, organizar e, prin- cipaimente, transformar essa informação em conhecimento novo4. Para Lima et al. 5 , devido ao desenvolvimento tecrológico, o conhecimento vai se tornando obsoleto, exigindo do profissional de saúde uma atitude contínua de aprender e habilidades para a busca e crítica das informaçōes obtidas.

Almeida $a^{b}$ afirma que, no que se refere às estratégias educacionais aplicadas à medicina, a metodologia de aprendizagem baseada em problemas vern se fortalecendo nos paises da América Latina, pois consegue introduzir maior eficiência nas práticas de ensino-aprendizagem e formar profissionais mais críticos e mais bem preparados para a auto-aprendizagem ao longo do exercício da profissão. A Faculdade de Medicina de Marília, a partir de 1997, introduziu pioneiramente no Brasil a metodologia ABP em seu curso de Medicina.

Segundo Komatsu', essa metodologia se apóia em quatro concepções de ensino-aprendizagem: aprendizagem autodirigida, baseada em problemas, em pequenos grupos de tutoria e orientada para a comunidade. $\mathrm{O}$ mesmo autor afirma que a busca e a aquisiçăo de conhecimentos constituem um processo contínuo ao longo da vida de cada indivíduo. Para isto, nessa nova metodologia, os estudantes săo encorajados a definir seus objetivos de aprendizagem e a assumir responsabilidade por avaliar seus progressos pessoais, tomando como parâmetros os objetivos formulados a cada unidade educacional. Menita et al. ${ }^{8}$ a firmam que, na metodologia $A B P$, o estudante é livre e responsável pela escolha das fontes de informação ou dos recursos educacionais que comporão seu aprendizado.

A residência médica foi implantada no Brasil em 19451946 pelo Hospital das Clínicas da Faculdade de Medicina da Universidade de São Paulo, seguindo o modelo americano, que já possuía programas de treinamento/aprendizado para pós-graduados em Medicina desde 1889 e constitui o mais recomendável programa para a formação do especialista. Nele, o médico recém-formado deverá se aperfeiçoar e trabalhar em serviços hospitalares de áreas específicas, sob a orientaçăo de professores ou de outros médicos de reconhecida experiência e competência ${ }^{3}$.

Em 1977, foí criada a Comissão Nacional de Residência Médica (CNRM), que regulamenta a prática da residência médica no Brasil. Atuaimente, a residência médica é pré-requisito obrigatório para a pós-graduação stricto sensu, segundo o Consetho Federal de Educação".

Segundo Feuerwerker $1,70 \%$ dos médicos que se formam no Brasil freqüentam um programa de residência médica, em que as principais funções são: complementação da graduaçâo, especialização e melhor preparo para inserção no mercado de trabalho. 
Na Faculdade de Medicina de Marília, a residência médica foi credenciada a partir de 198210.

Os residentes constituem uma relevante parcela de usuários da Biblioteca da Famema, que, nos últimos anos, oferece cursos de capacitaçāo em buscas de informaçð̌es a seus usuários, principalmente docentes e discentes. Para os residentes, as cursos são oferecidos de acordo com as solicitações de algumas especialidades.

Como ferramenta para melhor planejamento de seus serviços, a biblioteca deve realizar estudos das necessidades informacionais e dificuldades encontradas pelos usuários ที recuperação e seleção dos documentos. Estudos de usuários auxiliam a biblioteca na previsão da demanda ou na mudança da demanda de seus produłos c serviços, permitindo adequálos a cada época. A maioria desses estudos teve inicio na segunda metade da década de 194011,12. Figueiredo" afirma que o que se observa foi uma mudança de atitude em relação aos usuários. Até entāo, aguardava-se que eles se dirigissem às bibliotecas para buscar informaçōes, e a partir dessa época as bibliotecas passaram a antecipar as necessidades dos usuários, oferecendo-lhes serviços disponiveis para uso.

Atualmente, refletindo sobre esse contexto, podemus observar outra realidade, não diferente das citadas acima, mas que as engloba, pois hoje os usuários buscam a informação desejada em várias fontes e de várias maneiras, e a maioria das bibliotecas tem dificuldades em atender essas demandas de forma satisfatória. Estudos de usuários continuam como uma necessidade e carregam as prioridades dos anteriores, não mais apenas para oferecer um serviço novo e "resgatar" usuários, mas também para melhor atendê-los em suas necessidades já explicitadas.

Curty ${ }^{13}$ relaciona vários aspectos que permitem verificar a importância dos estudos de usuários, dos quais destacamos alguns que expressam os objetivos de nosso estudo: identificação do uso de recursos de informação; identificaçāo de comportamentos e hábitos dos usuários quanto ao uso e busca de infomaçōes; identificação das necessidades de qualificaçāo de determinada categoria de usuários; e identificação dos fatores que determinam o uso ou não das fontes de informaçāo.

A educação de usuários, segundo Belluzzo 14, é o processo pelo qual eles devem interiorizar compurtamentos adequados de uso de bibliotecas e desenvolver habilidades de interaçåo permanentes com os sistemas de informaçāo.

Dudziak et al ${ }^{15}$ afirmam que a educação de usuários rećne várias ferramentas, como instrução, treinamento, apresentação de interfaces amigáveis, manuais, tours, cursos de acesso a bases de dados, orientação bibliográfica, entre outras. Esses mesmos autores sustentam que a educaçảo de usu- ȧrios engloba várias aprendizagens c se constitui em aprender a aprender, aprender a pensar e a ser um usuário eficiente da informação, aprendendo a identificar, buscar, localizar, avaliar e selecionar a informação adequada para cada necessidade.

Devidu aos avanços de nuvas tecnologias aplicactas ao armazenamento e recuperação da informação, permitindo o aumento de documentos publicados eletronicamente, disponibilidades e acesso a bases de dados e catálogos automatizados de bibliotecas, várias bibliotecas no Brasil passaram a oferecer cursus de capacitação a seus usuários, conforme relatado na literatura mais recente $8,15-14$.

\section{METODOLOGIA}

A população-alvo estudada foi constituida por médicos residentes de Medicina da Faculdade de Medicina de Marilia, matriculados no Programa de Residência Médica da instituiçāo em 2003, distribuídos entre 10 ano (50 vagas, sendo 10 no Programa Saúde da Familia), 2e ano (33 vagas), 32 ano (14 vagas) e 40 ano (8 vagas), perfazendo um total de 105 sujeitos.

A coleta de dados foi realizada por meio de questionário, que permitiu investigar os sujeitos da pesquisa quanto a suas experiências em uso de fontes de informação, enfocandu a capacitação de usuários em busca de informaçōes durante a graduaşão em Medicina. O questionário foi constituido de questōes fechadas, algumas com opções de múltipla escolha, bem como possibilidade de justificativas em alguns casos.

Quanto à aplicaçāo do questionário, todos os residentes do 10 ano responderam ao mesmo durante uma reuniāo, na qual $90 \%$ estavam presentes. Os demais (25 ao to ano) receberam o questionário por intermédio das secretarias de cada especialidade e tiveram um prazo de 20 dias para devolvê-los.

Os dados coletados foram analisados quantitativa e qualitativamente, no intuito de atingir os objetivos propostos.

\section{RESULTADOS}

Dos 105 questionários distribuidos, 77 (73,33\%) retornaram e foram considerados para análise.

Com relaçāo à metodnlogia de ensino adotada, 17 alunos (22\%) foram graduados pela metodologia ABP e 60 alunos (78\%) pela metodologia tradicional de ensino médico.

Quanto ì experiência de uso de bibliotecas automa tizadas ou nāo, durante a graduaçāo em Medicina, 58 residentes $(75 \%)$ indica ram ter experiência com bibliotecas a utomatizadas e 19 $(25 \%)$ somentc com catálogos manuais de bibliotecas.

No que diz respeito ao acesso físico ao material, 66 residentes $(86 \%)$ responderam ter experiéncias no uso de bibliotecas com acervos abertos e $11(14 \%)$ com acervos fechados. 
A Tabela 1 mostra os residentes que tiveram capacitaçāo/ orientaçāo para uso de bibliotecas durante a graduaçāo em Medicina.

Sessenta e oito residentes ( $88 \%$ ) tiveram indicaçāo de literatura pelos docentes para seus estudos curriculares durante a graduaçāo, e apenas 9 (12\%) não tiveram. A maioria (60\%) a considerou suficiente para os estudos.

A Tabela 2 mostra o resultado quanto ao uso de fontes de informação e bases de dados bibliográficas.

Com relação ao uso de $e$-mail pelos residentes, observa-se que somente 6 deles ( $8 \%$ ) ainda não possuem cadastro nesses serviços.

No que se refere aos apontamentos de dificuldades ou nāo em buscas e localizaçāo de informaçāo para seus estudos

TABELA 1

Residentes que tiveram capacitação/orientação para uso de bibliotecas durante a graduação em Medicina

\begin{tabular}{l|c|c}
\multicolumn{1}{c|}{ Capacitação/Orientação } & Total & $\%$ \\
\hline Cursos de capacitação & 10 & 13 \\
\hline Visita orientada & 16 & 21 \\
\hline Visita orientada e cursos de capacitaçāo & 25 & 32 \\
\hline Nenhuma das alternativas & 26 & 34 \\
\hline
\end{tabular}

TABELA 2

Uso de fontes de informação e bases de dados bibliográficas por residentes durante a graduação em Medicina

\begin{tabular}{l|c|c}
\hline \multicolumn{1}{c|}{ Fontes } & Total & $\%$ \\
\hline Livros e folhetos & 76 & 98,70 \\
\hline Revistas impressas & 66 & 85,71 \\
\hline Medline & 59 & 76,62 \\
\hline Sites gerais na internet & 52 & 67,59 \\
\hline Apostilas & 44 & 57,14 \\
\hline Lilacs & 43 & 55,84 \\
\hline Cochrane & 33 & 42,86 \\
\hline Outras bases da Bireme & 31 & 40,26 \\
\hline Anais de eventos & 25 & 32,46 \\
\hline CD-ROMs & 23 & 29,88 \\
\hline Slides & 19 & 24,67 \\
\hline Fitas de videocassete & 16 & 20,78 \\
\hline Teses e dissertaçōes & 14 & 18,18 \\
\hline SciELO & 8 & 10,38 \\
\hline Outras fontes & 6 & 7,79 \\
\hline Teses e dissertaçỏes eletrônicas & 4 & 5,19 \\
\hline Ovid & 2 & 2,60 \\
\hline ProBE & 2 & 2,60 \\
\hline
\end{tabular}

curriculares, verificamos que 51 residentes (66\%) indicaram não ter encontrado dificuldades, e 26 (34\%) sim.

\section{DISCUSSÃO}

Considerando nossos objetivos iniciais e partindo do pressuposto de que as habilidades na recuperaçāo, seleção e uso das informaçōes se destacam cada vez mais como itens necessários à formação dos profissionais médicos, devendo, portanto, ser conteúdo oferecido aos estudantes de Medicina desde a graduação, destacamos alguns achados relevantes de nossa pesquisa. Lembramos que nossos estudos foram realizados tendo como sujeitos residentes em Medicina. Contudo, os questionamentos se referem à aquisiçāo de habilidades em busca de informaçōes durante a graduação, com o objetivo de avaliar as necessidades e experiências relativas ao assunto.

A distribuição dos sujeitos entre residentes graduados pela metodologia de ensino médico tradicional (78\%) e $\mathrm{ABl}^{3}(22 \%)$ já cra esperada, por ser a Famema a primeira escola médica no Brasil a implantar a metodologia ABP, sendo que cm 2002 tivemos a conclusão do curso pela primeira turma. Dessa forma, nāo contamos ainda com dados em literatura que comente essa distribuição.

A ıлаioria (75\%) dos médicos recém-formados já possui experiências de uso de bibliotecas com catálogos automatizados durante a graduação. Entre esses, (21\%) afirmaram ter convivido com ambas as formas (catálogos automatizados e manuais), o que deduzimos que sejam bibliotecas em processo de automação. Este resultado é compatível com a previsāo feita por Cunha ${ }^{20}$, segundo a qual quase todas, senão todas as bibliotecas universitárias brasileiras estarāo automatizadas até o ano 2010. Entretanto, neste estudo, por se tratar de bibliotecas universitárias específicas da área médica, consideramos alto o indice ( $25 \%$ ) de médicos graduados recentemente por escolas cujas bibliotecas ainda trabalham somente com catálogos manuais.

Durante a graduação em Medicina, somente $14 \%$ dos residentes conviveram com bibliotecas cujos acervos eram fechados. Para nosso estudo, consideramos importante esse resultado (maioria com acervo aberto), pois esta questz̄o foi abordada por entendermos que a independência do usuário na busca e seleção de informaçōes deverá ser completa, incluindo também a localização física dos documentos.

Quanto à capacitação em buscas de informaçōes (Tabela 1), item que consideramos importante para a construção do novo perfil médico, menos da metade (45\%) dos residentes apontaram ter sido capacitados por meio de cursos durante a graduação) em Medicina (cursos de capacitação: 13\%; visita orientada e cursos de capacitação: $32 \%$ ). A literatura mais atu- 
al recomenda que as bibliotecas universitárias brasileiras passem a trabalhar para a auto-suficiência ou auto-independência de seus usuários, como apontam Dudziak et al.15, Cuen$\mathrm{ca}^{16}$, Marquetis et al. ${ }^{18}$, Cunha ${ }^{20}$ e Lima e Souza ${ }^{21}$, entre outros. Percebemos, porém, que na literatura ainda são poucas as experiências relatadas sobre a capacitação em buscas de informaçōes específicas para alunos de graduação. Entre elas, estão os trabalhos de Menita et al. ${ }^{8}$, Moser e Accetta ${ }^{17}$ e Fernandes et al.22. Esse fato talvez esteja ocorrendo por se tratar de um serviço que vem sendo implantado nos últimos anos nas bibliotecas brasileiras dando prioridade a grupos de usuários que "aparentemente" estariam mais envolvidos com pesquisas, como docentes e alunos de pós-graduação. Ressaltanos que todos os graduados pela metodologia ABP foram capacitados para busca de informações por meio de visitas orientadas e cursos, num trabalho conjunto da biblioteca e da disciplina de informática em saúde (Famema), e somente um estudante $(5,88 \%)$ declarou ter tido dificuldades em buscar informações para seus estudos curriculares.

Na metodologia de ensino médico tradicional, normalmente os estudantes contain com indicação de literatura para seus estudos por parte dos docentes. Portanto, como em nosso estudo houve maior incidência de residentes graduados pela metodologia de ensino tradicional, houve também alta incidência ( $88 \%$ ) de residentes que tiveram indicação de literatura para seus estudos curriculares durante a graduação. Esse achado é confirmado por Peixoto e $\operatorname{Matos}^{23}$, que investigaram as fontes de estudos em escola médica na Região Sudeste do Brasil e relataram o fornecimento de listas bibliográficas como o principal tipo de orientação para estudos oferecido aos estudantes pelo conjunto de docentes. Na Famema, não ocorre indicação de literatura para estudos, existem sugestōes de sites e/ou documentos que serviram de base para a construção das unidades educacionais. Mesmo assim, 7 estudantes graduados pela metodologia ABP (41\%) consideraram ser suficiente a indicação de literatura para estudos; os demais responderam não ter indicação e/ou não ser suficiente para seus estudos. Esse resultado requer estudos mais específicos para avaliação, uma vez que o método é centrado no aluno e enfatiza a responsabilidade dele pelo próprio aprendizado, inclusive na busça de informações.

Como esperado, $60 \%$ dos sujeitos apontaram que a indicação de literatura recebida pelos docentes foi suficiente para seus estudos curriculares, pois, na metodologia tradicional de ensino médico, a aprendizagem é centrada no professor, com transmissão passiva do conhecimento.

Quanto ao uso de fontes de informação e bases de dados bibliográficas (Tabela 2), alguns itens se referem ao suporte da informação, como CD-ROMs (a informação armazenada pode ser uma base de dados bibliográfica, um livro, um periódico, etc.) ou SciELO e ProBE, por exemplo, que dão acesso a periódicos eletrônicos. Itens como esses foram inseridos em nossa investigaçāo porque objetivamos conhecer as habilidades dos sujeitos da pesquisa quanto ao uso de fontes e recursos informacionais.

Livros e folhetos $(98,70 \%)$, periódicos impressos $(85,71 \%)$ e a base de dados Medline $(76,62 \%)$ aparecem como as mais usadas pelos residentes enquanto alunos de graduação em Medicina. Nos estudos de Peixoto e Matos ${ }^{23}$, Rankin ${ }^{24}$ e Puga ${ }^{25}$, os livros também foram citados como a principal fonte de estudos por estudantes de Medicina. No estudo de Rankin ${ }^{24}$, como em nossa pesquisa, o periódico foi citado em segundo lugar, e a base de dados Medline em terceiro.

As buscas em sites gerais na internet aparecem em quarto lugar em nosso estudo (52\%). Por se tratar de fontes utilizadas por meio dos novos recursos aplicados à tecnologia da informação, consideramos bons esses índices de apontamentos. No estudo de Puga ${ }^{25}$, diferentemente do nosso, a internet aparece em segundo lugar.

Em nosso estudo, as apostilas foram fontes utilizadas por $57,14 \%$ dos residentes durante a graduação em Medicina. Esse resultado ficou um pouco abaixo do esperado, pois é bastante cornum utilizar essa fonte para estudos na metodologia de ensino tradicional. No estudo de Peixoto e $\mathrm{Matos}^{23}$, as apostilas aparecem em terceiro lugar, apontadas por $70 \%$ dos sujeitos investigados. Nesse mesmo estudo, as anotações de aula (própria) aparecem em segundo lugar (80\%). Em nosso estudo, esse item não foi abordado e foi citado na opção "outras fontes" em baixa porcentagem, sem significância.

A base de dados Lilacs foi apontada por mais da metade dos sujeitos investigados $(55,84 \%)$. Mesmo tendo boa utilizaçăo, este índice talvez possa ou deva ser melhorado por meio de capacitação, pois a consideramos uma fonte valiosa e imprescindivel à área médica, urna vez que é principalmente através dela que recuperamos os artigos científicos publicados em periódicos nacionais e da América Latina da área de ciências da saúde, além de outros documentos. No estudo de Puga ${ }^{25}$, a base de dados Lilacs aparece em quarto lugar entre as fontes utilizadas por estudantes de Medicina, năo diferindo muito do nosso resultado, pois aquele estudo nảo aborda o item apostilas, que, no nosso caso, foi mais utilizado do que a base Lilacs.

Observamos que houve inversão de uso entre as bases Lilacs e Medline se compararmos nosso estudo com a pesquisa de Puga ${ }^{25}$, pois em nosso estudo a base de dados Medline foi mais usada do que a Lilacs. Não abordamos a questāo da 
barreira lingüística, porém observamos que a base de dados Medline, em inglês, teve alto índice $(76,62 \%)$, fazendo crer que, entre os sujeitos estudados, poucos apresentam dificuldades com aquele idioma.

A Cochrane foi apontada por $42,86 \%$ dos sujeitos investigados, resultado que consideramos além do esperado, por se tratar de uma fonte específica de documentos voltados para estudos de medicina baseada em evidências, que requer do usuário um conhecimento maior sobre análise da qualidade da documentação médica. Por se tratar de uma fonte de grande valor e qualidade para a área médica, acreditamos que ela deva fazer parte do conteúdo de cursos de capacitação para usuários da área. Em nosso estudo, verificamos que $60 \%$ dos residentes capacitados para buscas de informações durante a graduação em Medicina, por meio de cursos, indicaram ter usado a Cochrane como fonte de informaçỏes para seus estudos curriculares.

Na Famema, a medicina baseada em evidências faz parte do conteúdo curricular da medicina, e todos os estudantes são capacitados para o uso da Biblioteca Cochrane. As opções: outras bases de dados da Bireme; anais de eventos; CD-ROMs; slides; fitas de videocassete; teses e dissertações tiveram menos de $50 \%$ de indicação de uso. Os itens CD-ROMs ( $29,88 \%$, slides $(24,67 \%)$ e fitas de videocassete $(20,78 \%)$ foram também destacados pela pesquisa de Puga ${ }^{25}$ como pouco utilizados. A mesma autora aponta a importância de as bibliotecas estimularem o uso desses materiais que consideramos de boa qualidade para os estudos. A SciELO foi pouca utilizada pelos sujeitos de nossa pesquisa, apenas $10,38 \%$ a usaram. As teses e dissertações eletrônicas foram usadas por apenas 5,19\% dos sujeitos investigados. Observamos que as teses tiveram baixo índice de uso, mesmo na forma impressa $(18,18 \%)$. Ovid e ProBE tiveram o mesmo índice de utilização $(2,60 \%)$ e foram abordados em nosso estudo, conforme apontado acima, para investigar a familiaridade dos sujeitos com os recursos tecnológicos aplicados à informação. No entanto, o pouco uso ou desconhecimento dos mesmos já era esperado, por se tratar de recursos de alto custo e ainda não disponíveis na maioria das bibliotecas. Constatamos também que somente 7 residentes $(9,10 \%)$ usaram apenas fontes de informações impressas, como livros, folhetos, periódicos e apostilas. Nessa população, todos apontaram não ter tido cursos de capacitação, e somente 1 teve visita orientada à biblioteca.

O cadastro em serviços de e-mail (correio eletrônico) teve alto índice de apontamento pelos residentes: apenas $8 \%$ deles ainda não o possuem. Esse item também foi investigado em nosso estudo com o objetivo de avaliar a familiaridade dos sujeitos com os recursos de informática e por ser um meio de comunicação amplamente usado para transferência de informaçōes, portanto de grande utilidade para esses profissionais. Carvalho Junior ${ }^{26}$ et al. afirmam que o uso do correio eletrônico pode ser tomado como um elemento representativo dos serviços de internet em geral. Em seu estudo, que compara a utilização de correio eletrônico entre estudantes ingressantes no curso médico da Faculdade de Medicina de Marília entre os anos 1997, 1998 e 2000, apontam o crescente indice de estudantes que já possuem cadastro em serviços de $e$-mail ao ingressarem na faculdade.

Com relação aos apontamentos para dificuldades ou năo na busca de informações para seus estudos curriculares, enquanto alunos de graduação, $66 \%$ dos residentes indicaram "não" e 34\% "sim". Estes resultados, se analisados apenas quantitativamente, discordam de nossas afirmativas de que, para não terem essa dificuldade, os usuários devem ter capacitação/orientação de uso de bibliotecas. Esta nossa afirmação parte de nossas observações práticas profissionais e dos achados da literatura, que afirmam que as bibliotecas devem capacitar seus usuários para a auto-suficiência em buscas de informaçő̉es $8,16,17,18,22$. Destacamos que, nesta questão, demos a oportunidade de os sujeitos justificarem suas respostas, e 22 $(84,61 \%)$ daqueles que responderam "sim" fizeram justificativa. Dos que responderam "não", apenas $15(29,41 \%)$ justificaram. A maioria das justificativas tanto para "sim", como para "não", menciona a capacitação/orientação em busca de informações ou uso de bibliotecas como fator determinante da dificuldade/facilidade. Além disso, quando analisamos o conjunto dos resultados da pesquisa e as justificativas fornecidas para a questão, percebeinos que a capacitação se faz necessária em maior escala, pois tivemos médio e baixo índice de utilização para várias fontes que pođeriam estar sendo utilizadas por esses estudantes. Como já abordamos, a maioria dos sujeitos investigados foi graduada pela metodologia de ensino médico tradicional, na qual ocorre indicação de literatura, e a maioria deles a considerou suficiente, eliminando, portanto, a necessidade de busca de outras informações. Vale lembrar também que nosso objetivo é avaliar as habilidades em buscas de informações por médicos recém-formados, tendo como parâmetros o novo perfil médico exigido e os avanços tecnológicos aplicados à informação.

Outro objetivo de nosso estudo foi verificar se existem diferenças entre médicos graduados pela metodologia de ensino tradicional e ABP com relação a buscas de informações. Concluímos que sim, pois, entre os formados pela metodologia $\mathrm{ABl}$, todos capacitados, somente 1 deles $(5,88 \%)$ apontou ter tido dificuldade para buscas de informaçōes durante a graduação. A utilização de diferentes fontes de informação foi 
maior também pelos residentes graduados pela metodologia $A B P$. Esse achado foi também apontado por Puga ${ }^{25}$, que afirma que estes utilizam fontes variadas devido à necessidade de auto-aprendizagem e conhecem mais os recursos disponíveis na biblioteca. A mesma afirmação é encontrada em Rarkin ${ }^{24}$, que sustenta que os graduandos em Medicina pela metodologia ABP relatam maior facilidade no uso dos recursos informacionais em comparação àqueles que se graduaram pela metodologia tradicional de ensino médico.

\section{CONCLUSÃO}

Mudanças no perfil do profissional médico e avanços tecnológicos aplicados à informação provocam mudanças também nos recursos e serviços oferecidos pelas bibliotecas. Dessa forma, as bibliotecas de medicina ou ciências da saúde deverão conhecer as novas necessidades dos usuários e prepará-los para o novo desafio, capacitando-os e fornecendo-lhes o suporte necessário.

Consideramos também que, diante da expressiva e crescente produção da informação médica, estes profissionais necessitam cada vez mais dominar as técnicas de busca e seleção das informações necessárias às suas práticas profissionais. Portanto, sugerimos que a capacitação dos usuários para a auto-suficiência no uso de bibliotecas e informações seja oferecida a todos os usuários, independentemente da metodologia de ensino ou do nível de graduação.

Os resultados da pesquisa, de forma geral, mostram que estamos passando por uma fase de transição de perfil desses profissionais quanto à capacitação específica para buscas de informações. Muitas bibliotecas de escolas médicas já estão incluindo essa atividade em seus serviços.

Nossos resultados sugerem também a predominância de médicos recém-formados que "parecem" satisfeitos com o aprendizado passivo. Este achado já era esperado e não condiz com as atuais exigências de perfil desses profissionais. Estes devem ser educados para a auto-aprendizagem. No caso do uso de informações, isto significa adquirir independência própria na busca, seleção e utilização de informações para solução de problemas do dia-a-dia. Portanto, ressaltamos que as bibliotecas pertencentes a instituiçōes que oferecem serviços de residência médica devem estender o serviço de capacitação/orientação de usuários também aos médicos residentes, como uma forma de sanar as dificuldades daqueles que ainda não contaram com esses serviços durante a graduação.

Sabemos que a área de ciências da saúde tem sido sempre uma das mais bem organizadas e disponibilizadas no que diz respeito à documentação científica, em âmbito tanto nacional como internacional. Hoje, ela dispõe de recursos e fontes in- formacionais extremamente valiosos, e, portanto, a falta de conhecimento ou de orientação de seus usuários não deverá ser fator que impeça seu uso.

Frente aos resultados, a biblioteca da Famema, em conjunto com a Comissão de Residência Médica da instituição, oferecerá cursos de capacitação para todos os seus médicos residentes (1s ao 40 ano). A opção de oferecer o curso a todos se deve principalmente à necessidade de capacitação para uso de nossas bases de dados internas $\mathrm{e}$ ao resultado que aponta baixo uso de algumas bases de dados que consideramos importantes para a árca. Na prática, sabemos que muitos usuários julgam saber usar determinadas fontes de informação e descobrem, durante o curso/orientação, que não as estavam usando adequadamente. Mesmo assim, acreditamos que alguns residentes poderão eliminar alguns itens do conteúdo do curso de acordo com suas necessidades, segundo aponta nossa pesquisa.

Os estudantes de graduação e docentes dos cursos de Enfermagem e Medicina e estudantes dos cursos de especializaçăo oferecidos pela Famema contam com cursos de capacitação em busça de informações oferecidos pela biblioteca desde 1997, num trabalho em parceria com a disciplina de informática em saúde e unidades educacionais. Por solicitaçâa de algumas especialidades, alguns residentes também já estão sendo capacitados pela biblioteca nos últimos anos, serviço que pretendemos estender a todo o grupo, como afirmado acima. A biblioteca continua a trabalhar constantemente com seus usuários já capacitados no sentido de auxiliá-los no esclarecimento de dúvidas, levantamentos bibliográficos que exijam maior conhecimento de vocabulários controlados ou refinamentos de pesquisa, apresentaçăo de interfaces novas de recuperação de informaçōes e serviços novos, entre outros.

\section{AGRADECIMENTOS}

Ao Prof. Dr. José Bitu Moreno, docente da Famema e presidente da Coreme em Marília; a Regina Helena Gregório Menita, bibliotecária chefe da Famema; e à Prof̣ Dra Mariângela Spotti Lopes Fujita, docente na Unesp/Marília e orientadora deste trabalho, pelo incentivo e colaboração na execuçāo desta pesquisa.

\section{REFERENCIAS}

1. Feuerwerker LCM. Mudanças na educação médica e residência médica no Brasil. São Paulo: HUCITEC; 1998.

2. Venturelli $]$. Os aspectos educacionais na reforma da educação nas profissões da saúde. In: Almeida $M$, Feuerwerker $\mathrm{L}$, Llamos CM, org. A educação dos profissionais de saúde na América Latina: teoria e prática de um movimento de mudança. São Paulo: HUCITEC; 1999. p.145-64. 
3. Lima-Gonçalves E. Médicos e ensino da medicina no Brasil. São Paulo: EDUSP; 2002. p.59-108.

4. Masetto MT. Discutindo o processo ensino/aprendizagem no ensino superior. In: Marcondes E, Lima-Gonçalves E, coord. Educação médica. São Paulo: Sarvier; 1998. p.11-9.

5. Lima VV, Komatsu RS, Padilha RQ. Desafios ao desenvolvimento de um currículo inovador: a experiência da Faculdade de Medicina de Marília. Interface: Comun Saúde Educ 2003: 7(12): 175-84.

6. Almeida MJ. Educação médica e saúde: limites e possibilidades das propostas de mudança. [tese] São Paulo, Faculdade de Saúde Pública, Universidade de São Paulo, 1997.

7. Komatsu RS, coord. Guia do processo de ensino - aprendizagem "aprender a aprender". $2^{\mathrm{a}}$ ed. Marilia (SP): Faculdade de Medicina de Marília; 1998.

8. Menita RHG, Lima HMC, Faria JB. Relato de experiência da biblioteca da Faculdade de Medicina de Marília (FAMEMA) na metodologia de aprendizagem baseada em problemas (ABP). Anais do $3^{\circ}$ Simpósio Intemacional de Biblioteconomia "Prof. Dr. Paulo Tarcísio Mayrink"; 1-3 set. 1999; Marília (SP): UNESP; 1999. p.67-73.

9. Bacheschi LA. A residência médica. In: Marcondes E, LimaGonçalves E, coord. Educação médica. São Paulo: Sarvier; 1998. p.369-73.

10. Faculdade de Medicina de Marília. Manual da residência médica. Marília (SP): FAMEMA; 2002.

11. Figueiredo N. Avaliação de coleções e estudo de usuários. Brasília (DF): Associação dos Bibliotecários do Distrito Federal; 1979. p.77-96.

12. Figueiredo NM. Estudos de usuários como suporte para planejamento e avaliação de sistemas de informaçăo. Cienc Inf $1985 ; 14(2): 127-35$.

13. Curty MG. Busca de informação para desenvolvimento das atividades acadêmicas pelos médicos docentes da UEM. Anais do $11^{\circ}$ Seminário Nacional de Bibliotecas Universitá-

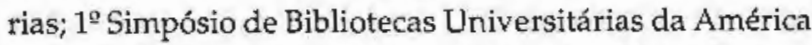
Latina e do Caribe; $1^{2}$ Simpósio de Diretores de Bibliotecas Universitárias da América Latina e do Caribe; 24-28 abr. 2000; Florianópolis: UFSC-BU. 1 CD-ROM.

14. Belluzzo RCB. Educação de usuários de bibliotecas universitárias: da conceituação e sistennatização ao estabelecimento de diretrizes. [dissertação] São Paulo, Escola de Comunicaçöes e Artes, Universidade de São Paulo, 1989.

15. Dudziak EA, Gabriel MA, Villela MCO. A educação de usuários de bibliotecas universitárias frente à sociedade do conhecimento e sua inserção nos novos paradigmas educaci- onais. Anais do $11^{2}$ Seminário Nacional de Bibliotecas Universitárias; $1^{\circ}$ Simpósio de Bibliotecas Universitárias da América Latina e do Caribe; $1^{\circ}$ Simpósio de Diretores de Bibliotecas Universitárias da América Latina e do Caribe; 24-28 abr. 2000; Florianópolis: UFSC-BU. 1 CD-ROM.

16. Cuenca AMB. Usuário da busca automatizada: avaliação do curso MEDLINE/LILACS no contexto acadêmico. [dissertação] São Paulo, Faculdade de Saúde Pública, Universidade de São Paulo, 1997.

17. Moser EM, Accetta IR. Acesso às bases de dados online: rotina de treinamento para usuários da Biblioteca da Biblioteca Central da FURB. Anais do $12^{\circ}$ Seminário Nacional de Bibliotecas Universitárias; 21-25 out. 2002; Recife: UFPE, 2002. Disponível em: http://www.acd.ufrj.br/sibi/snbu/ snbu2002/oral.pdf/62.a.pdf. Acesso em: 26 maio 2003.

18. Marquetis EM, Ribeiro CM, Lucas CR, Santos GC, Vicente $\mathrm{G}$, Cocconi HM et al. Avaliação do programa de capacitação de usuários do sistema de bibliotecas da UNICAMP. Anais do $12^{\circ}$ Seminário Nacional de Bibliotecas Universitárias; 21-25 out. 2002; Recife, UFPE, 2002. 1 CD-ROM.

19. Girello M. A utilizaçăo do serviço de treinamento e consulta às bases de dados pelos usuários da Biblioteca da Faculdade de Odontologia de Piracicaba - UNICAMP. Anais do $12^{2}$ Seminário Nacional de Biblitecas Universitárias; 21-25 out. 2002; Recife, UFPE, 2002. 1 CD-ROM.

20. Cunha MB. Construindo o futuro: a biblioteca universitária brasileira em 2010. Cienc Inf 2000; 29(1): 71-89.

21. Lima IF, Souza AB. Uso de bases de dados na biblioteca de pós-graduação em odontologia do CCS/UFPE. Anais do $12^{2}$ Seminário Nacional de Bibliotecas Universitárias; 21 25 out. 2002; Recife, UFPE, 2002. 1 CD-ROM.

22. Fernandes DMS, Aguiar, IM, Lima LR. Aprendizagem baseada em problemas - (PBL) e a Biblioteca Central da Universidade Estadual de Londrina. Olho Mágico 2000; 6(21); 16-7.

23. Peixoto MAP, Matos TM. Fontes de estudo na escola médica: recursos instrucionais utilizados por alunos de medicina da região sudeste do Brasil. Rev Bras Educ Med 2002; 26(1):28-34.

24. Rankin JA. Problem-based learnirg medical education: effect on library use. Bull Med Libr Assoc 1992: 80(1): 36-46.

25. Puga MES. Estudo das necessidades e habilidades de busca de informação de estudantes de medicina em metodologia pedagógica ABP - Aprendizagem Baseada em Problemas. 2000. Trabalho de Conclusão de Curso (TCC), apresentado ao Conselho de Curso de Biblioteconomia da UNESPMarília, como parte das exigências para a obtenção do título de Bacharel em Biblioteconomia. 
26. Carvalho Junior PM, Menita RHG, Carvalho VCL. Utilização do correio eletrônico por estudantes ingressantes no curso médico da Faculdade de Medicina de Marília: uma comparação entre os anos de 1997, 1998 e 2000. Anais do $38^{\circ}$ Congresso Brasileiro de Educação Médica; $5^{2}$ Fórum Nacional de Educação Médica; 18-22 set. 2000; Petrópolis, ABEM, 2000. p.124.

\section{Endereço para correspondência}

Rua Salvador Mendes de Almeida, 555 17580-000 - Pompéia - SP

e-mail: helenafamema@yahoo.com.br 